\title{
Thermal and Epithermal Neutron Fluence Rates in the Irradiation Facilities of the TRIGA IPR-R1 Nuclear Reactor
}

\author{
Dante Marco Zangirolami* \\ Centro de Desenvolvimento da Tecnologia Nuclear CDTN/CNEN, Av. Presidente Antônio Carlos, \\ 6627 - Campus UFMG, 31270-901, Belo Horizonte, MG, Brazil and \\ Departamento de Engenharia Nuclear DEN/UFMG, Av. Antônio Carlos, \\ 6627 - Campus UFMG, 31270-090 Belo Horizonte, MG, Brazil \\ Arno Heeren de Oliveira \\ Departamento de Engenharia Nuclear DEN/UFMG, Av. Antônio Carlos, \\ 6627 - Campus UFMG, 31270-090 Belo Horizonte, MG, Brazil \\ Andréa Vidal Ferreira \\ Centro de Desenvolvimento da Tecnologia Nuclear CDTN/CNEN, \\ Av. Presidente Antnio Carlos, 6627 - Campus UFMG, 31270-901, Belo Horizonte, MG, Brazil
}

(Received on 25 September, 2009)

\begin{abstract}
The TRIGA IPR-R1 nuclear reactor at the Centro de Desenvolvimento da Tecnologia Nuclear CDTN/CNEN, Belo Horizonte, Brazil, has three irradiation facilities: a rotary specimen rack outside the reactor core with 40 irradiation channels which provide large scale isotope irradiation; the central tube placed at the center of the reactor core which permits sample irradiation with maximum neutron flux, as well as neutron beam extraction; a pneumatic transfer tube which makes possible the analysis of short half-life radioisotopes. The aim of this work is to evaluate the thermal and epithermal neutron fluence rates in the three irradiation facilities of the IPR-R1. $\mathrm{Al}-0.1 \% \mathrm{Au}$ reference materials were irradiated bare and Cd-covered, at $100 \mathrm{~kW}$ thermal power, and gamma spectrometry was applied with an HPGe detector. For the rotary specimen rack, the values obtained for thermal and epithermal neutron fluence rates are $(8.2 \pm 0.2) \times 10^{11} \mathrm{n} . \mathrm{cm}^{-2} \cdot \mathrm{s}^{-1}$ and $(3.5 \pm 0.1) \times 10^{10} \mathrm{n} . \mathrm{cm}^{-2} . \mathrm{s}^{-1}$, respectively. For the pneumatic transfer tube and the central tube, epithermal neutron fluence rate values are respectively $(3.3 \pm 0.2) \times 10^{9} \mathrm{n} . \mathrm{cm}^{-2} . \mathrm{s}^{-1}$ and $(2.6 \pm 0.1) \times 10^{11} \mathrm{n} . \mathrm{cm}^{-2} . \mathrm{s}^{-1}$. For these facilities, thermal neutron fluence rate values are $(2.5 \pm 0.2) \times 10^{11} \mathrm{n} . \mathrm{cm}^{-2} . \mathrm{s}^{-1}$ and $(2.8 \pm 0.1) \times 10^{12} \mathrm{n} . \mathrm{cm}^{-2} . \mathrm{s}^{-1}$, respectively. Knowledge of these parameters will be fundamental in the planning of sample irradiation for analysis or radioisotope production in a more accurate way.
\end{abstract}

Keywords: TRIGA IPR-R1 nuclear reactor, neutron fluence rate, neutron activation analysis, irradiation facilities

\section{INTRODUCTION}

The IPR-R1 is a TRIGA Mark 1 reactor produced by General Atomics (USA). This reactor belongs to the Centro de Desenvolvimento da Tecnologia Nuclear CDTN/CNEN, located in the City of Belo Horizonte, Brazil, and has been operating since 1960 [1]. It is a pool type reactor cooled by natural circulation. The current (2009) core configuration has 63 fuel elements which consist of an alloy of zirconium hydride and uranium enriched to $20 \%$ uranium-235. Reactor power level is controlled by three control rods containing boron carbide. The principal instrumentation for the operation of the IPR-R1 TRIGA Mark 1 consists of four neutronic channels for measuring power, as well as channels for monitoring temperature, water conductivity, radiation level, etc. Figure 1 shows a diagram of the IPR-R1 pool and reactor core.

Figure 2 shows a horizontal cross-section of the actual reactor core configuration where it is possible to see the three irradiation facilities: the rotary specimen rack (RSR) outside the reactor core with 40 irradiation channels which provide large scale isotope irradiation; the central tube (CT) placed at the center of the reactor core which permits sample irra-

*Electronic address: dmzecdtn.br diation with maximum neutron flux, besides neutron beam extraction; the pneumatic transfer tube (PTT) which makes possible the analysis of short half-life radioisotopes. One of the main applications of the IPR-R1 is neutron activation analysis, NAA. Approximately 3000 samples are analyzed annually by this technique and, in most cases, samples are irradiated in the RSR.

The IPR-R1 core configuration has been changed six times. The original core configuration allowed steady-state operation at $30 \mathrm{~kW}[1]$. The current core configuration, from 2001, permits operations at a maximum steady-state power of $250 \mathrm{~kW}$ [2]. Our previous work [3] on the present core configuration evaluated the specific induced activity profile at the RSR and its variation under different irradiation conditions. For the actual core configuration, there are few experimental works concerning the characterization of the thermal and epithermal neutron fluence rates in IPR-R1 irradiation facilities. An experimental work [4] has evaluated the thermal and epithermal neutron fluence rates in five channels of the RSR. Thermal and epithermal neutron fluence rates in the PTT facility and epithermal neutron fluence rate in the CT facility have not been evaluated until now. Therefore, it is very important to evaluate and to update the data concerning the neutron fluence rates in IPR-R1 irradiation facilities. Knowledge of these parameters is fundamental to the planning of sample irradiation for analysis or radioisotope production. It permits calculation of neutron reaction rates in experiments, 


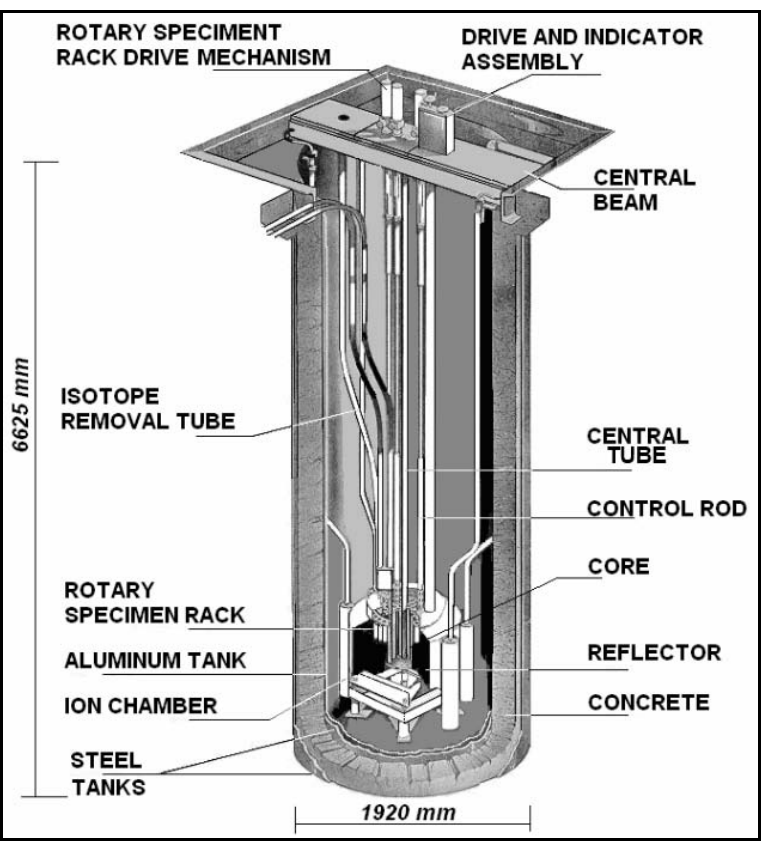

FIG. 1: The pool of the IPR-R1 TRIGA nuclear reactor (courtesy of Dr. A. Z. Mesquita).

enabling determination of experimental parameters such as specific induced activity, irradiation time, counting time, decay time, etc. The aim of the present work is to determine the thermal and epithermal neutron fluence rates in eight specific channels of the RSR, in the central position of the CT and in the PTT irradiation facilities of the TRIGA IPR-R1 nuclear reactor.

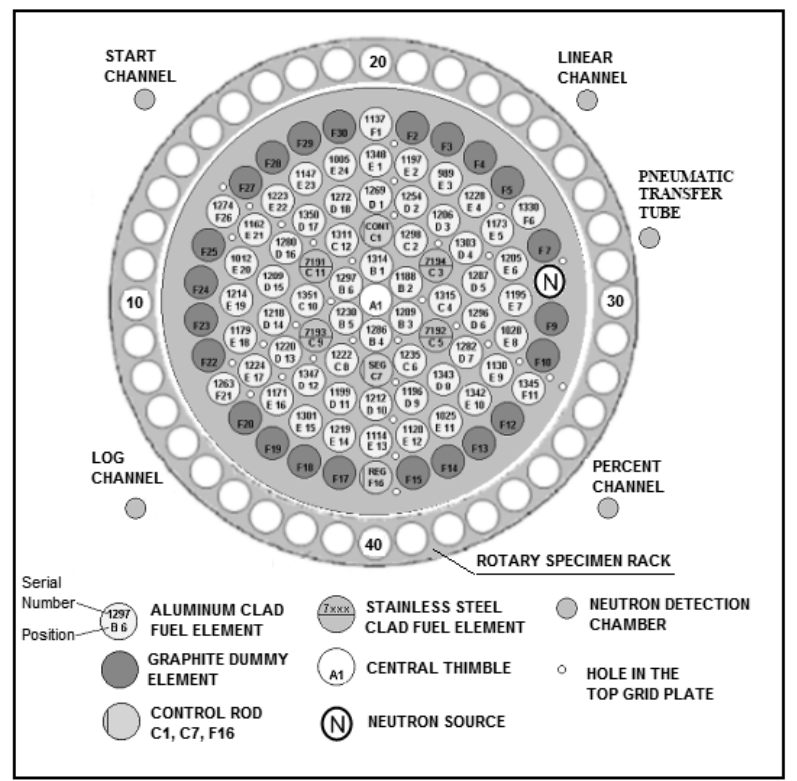

FIG. 2: Current horizontal cross-section of TRIGA IPR-R1 reactor core [3].

\section{MATERIALS AND METHODS}

The thermal and epithermal neutron rates in the IPR-R1 irradiation facilities were measured by the neutron activation method using bare and cadmium-covered foils [5]. Irradiations were performed between May and October, 2008. Al$0.1 \% \mathrm{Au}$ reference material [6] was used as the neutron fluence rate detector. The reference material was cut into disks about $1 \mathrm{~cm}$ in diameter and weighed on a precision balance. Each detector was approximately $23 \mathrm{mg}$. The experimental procedure for each of the irradiation facilities are described below. All the experiments were performed at $100 \mathrm{~kW}$ thermal power.

\section{Rotary Specimen Rack:}

Eight irradiation channels were chosen for the measurements: channels 5, 10, 15, 20, 25, 30, 35, 40. Previous work [3] had revealed that the specific induced activity of samples in the RSR has a fluctuation of approximately $2 \%$ for samples irradiated on different dates. In order to check variations of thermal and epithermal neutron fluence rates, bare and Cadmium-covered probes were irradiated in the selected irradiation channels on different dates. On any given date, reactor operational parameters, such as pool water temperature, control rod positions, etc., may present slight differences which reflect on induced activity in samples. In this experiment set, probes were irradiated for 1 hour. Another experiment was performed to determine thermal and epithermal neutron fluence rates in a single irradiation, in order to ensure the same reactor operating conditions. After reactor startup and power stabilization, sixteen probes were irradiated, one at a time, in the selected channels. Each probe was irradiated for 5 minutes. For each irradiation channel, two probes were used, one bare and one cadmium-covered. The reactor operation parameters remained stable during the experiment, which lasted approximately 3 hours.

Central Tube and Pneumatic Transfer Tube

For measuring CT and PTT neutron fluence rates, irradiations were performed on different dates. Bare and Cadmiumcovered probes were irradiated one at a time. In these experiments, probes were irradiated for 1 hour. For CT and PTT analysis, six and seven irradiations were performed respectively.

After neutron irradiation and appropriate decay time, probes were submitted to gamma spectrometry in a system equipped with an HPGe detector CANBERRA ${ }^{\circledR} 5019$ and Gennie 2000 v2.0, CANBERRA ${ }^{\circledR}$ software. The counting time was adjusted to provide a net peak area of at least 20,000 countings for the $411.8 \mathrm{keV}$ peak from the 198Au radioisotope. Gamma spectrometer analyses provide the induced activity of the probes at each irradiation.

The characteristic nuclear reactions in the experiments are:

$$
{ }^{197} A u(n, \gamma){ }^{198} A u
$$

$$
{ }^{27} \mathrm{Al}(n, \gamma){ }^{28} \mathrm{Al}
$$


${ }^{198} \mathrm{~A} u$ radioisotope has a half-life of 2.694 days, while the ${ }^{28} \mathrm{Al}$ radioisotope has a half-life of 2.24 minutes. Therefore, half an hour after ending irradiation, almost all ${ }^{28} \mathrm{Al}$ will have decayed without disturbing the ${ }^{198} A u$ counting.

Adopting the Högdahl convention [7], the sample induced activity $A_{0}$ at the end of the irradiation time $t_{i r r}$, is given by:

$$
A_{0}=N\left(\phi_{t h} \sigma_{t h}+\phi_{e p i} I_{\gamma}\right)\left(1-e^{-\lambda t_{i r r}}\right)
$$

where $N$ is the number of target nuclei in the sample; $\sigma$ th is the thermal neutron radioactive capture cross-section of the target nuclei; $\phi_{t h}$ and $\phi_{\text {epi }}$ are the thermal and epithermal neutron fluence rates, respectively, in the samples; $I \gamma$ is the radioactive capture resonance integral of the target nuclei; $\lambda$ is the radioisotope decay constant $\left(\lambda=\ln 2 / T_{1 / 2} ; T_{1 / 2}\right.$ is the radioisotope half-life). After gamma spectrometry analysis, the induced activity may be obtained by [7]:

$$
A_{0}=\frac{N_{p} e^{\lambda t_{d}}}{\varepsilon \gamma \theta w t_{m}}
$$

where $t_{d}$ is the time interval between the termination of irradiation and the start of counting; $t_{m}$ is the counting interval time; $\varepsilon$ is the photo peak counting efficiency; $\theta$ is the isotopic abundance of the target nuclide; $\gamma$ is the gamma emission probability; $w$ is the target nuclide mass in the sample; $N_{p}$ is the net peak area.

When samples are irradiated under Cadmium cover, the ( $\phi t h \sigma t h)$ term is much smaller than the $\left(\phi_{\text {epi }} \mathrm{I} \gamma\right)$ term, because Cadmium cover almost completely absorbs the thermal neutrons and transmits all the epithermal neutrons. Therefore equation (3) becomes:

$$
A_{0}=N\left(\phi_{e p i} I_{\gamma}\right)\left(1-e^{-\lambda t_{i r r}}\right)
$$

From equations (4) and (5), the neutron epithermal fluence rate $\phi_{\text {epi }}$ may be determined directly.

The $f$ parameter, defined as the thermal to epithermal neutron fluence rate ratio, is given by [6]:

$$
f=\frac{\phi_{t h}}{\phi_{e p i}}=Q_{0}\left(R_{C d}-F_{C d}\right)
$$

where $Q_{0}$ is the ratio of the radioactive capture resonance integral and the thermal neutron radioactive capture crosssection of the target nuclei; $R_{C d}$ is the Cadmium ratio, defined as the ratio of the activity of the bare sample to the activity of the Cadmium covered sample; $F_{C d}$ is the correction factor for Cadmium transmission of epithermal neutrons. When a bare and a Cadmium-covered sample are irradiated by a stable neutron flux in an irradiation channel, $Q_{0}, F_{C d}$ and $R C d$ are constants, experimentally determined, which permits determination of $\boldsymbol{f}$. Therefore, if the $\boldsymbol{f}$ parameter is known, the thermal neutron fluence rate is obtained by:

$$
\phi_{t h}=f \cdot \phi_{\text {epi }}
$$

\section{RESULTS AND DISCUSSIONS}

\section{$\underline{\text { Rotary Specimen Rack: }}$}

Figure 3 shows the results of specific induced activity on bare and cadmium-covered probes at different irradiations.

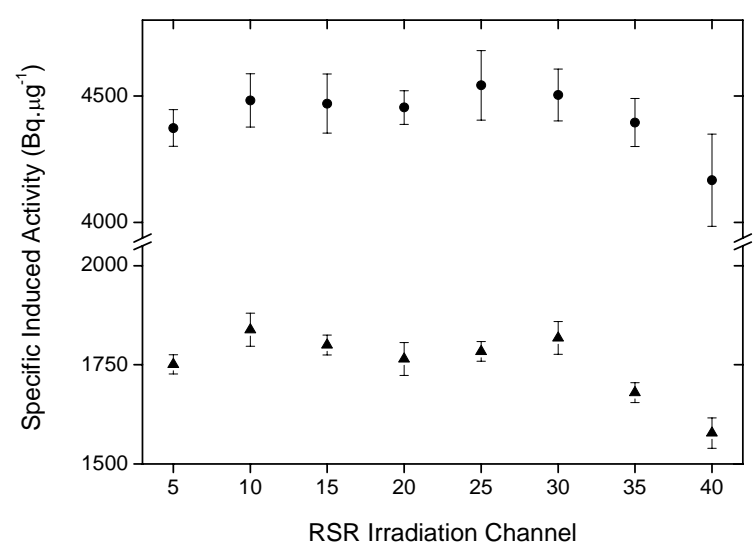

FIG. 3: Specific induced activity on bare (circles) and cadmiumcovered (triangles) probes irradiated in the TRIGA IPR-R1 Rotary Specimen Rack.

The results indicate a variation of $2.7 \%$ for values for induced activity on bare probes and a variation of $4.9 \%$ for values for induced activity on cadmium-covered probes. Results for bare samples are consistent with previous work [4], while the results for cadmium-covered probes were measured for the first time in this work. These results permit one to state that thermal and epithermal neutron fluence rates in the IPRR1 RSR have a fluctuation less than or equal to $5 \%$ in different reactor operations.

The obtained values for thermal and epithermal neutron fluence rates in the chosen RSR irradiation channels are shown in Figure 4.

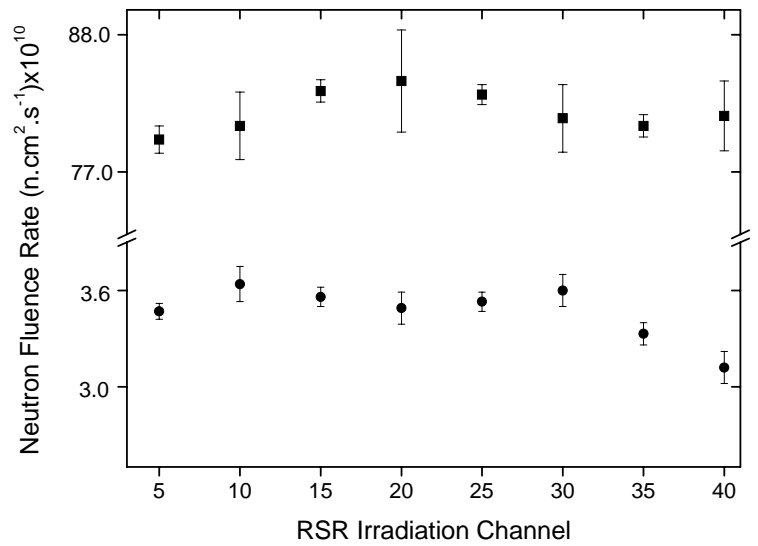

FIG. 4: Thermal (square) and epithermal (circle) neutron fluence rates at eight irradiation channels of the TRIGA IPR-R1 Rotary Specimen Rack.

The mean values and standard deviations for thermal and epithermal neutron fluence rates at the RSR are respectively $\phi_{t h}=\left(8.2 \times 10^{11} \pm 2 \%\right) n . \mathrm{cm}^{-2} \cdot \mathrm{s}^{-1}$ and $\phi_{\text {epi }}=\left(3.5 \times 10^{10}\right.$ $\pm 3 \%) \mathrm{n} . \mathrm{cm}^{-2} \cdot \mathrm{s}^{-1}$, which correspond to $f=24$. The values obtained in this work are respectively $30 \%$ and $20 \%$ higher than the previous results reported by other authors [4]. This difference may be due to the time lapse between the two works. The IPR-R1 reactor is periodically submitted to ther- 
mal calibration, the last one being carried out on March 5th, 2009 [8]. At that time, the value of the thermal power obtained by the heat balance method was $(112.0 \pm 6.6) \mathrm{kW}$. This value indicates that the difference between $100 \mathrm{~kW}$ nominal thermal power and the experimental power value may roughly reach $20 \%$, which explains our higher results when compared to those previous [4].

\section{Central Tube:}

The values obtained for thermal and epithermal fluence rates in the CT central position are: $\phi t h=\left(28.0 \times 10^{11} \pm 4 \%\right)$ n.cm ${ }^{-2} \cdot \mathrm{s}^{-1}$ and $\phi e p i=\left(26.0 \times 10^{10} \pm 4 \%\right) \mathrm{n} \cdot \mathrm{cm}^{-2} \cdot \mathrm{s}^{-1}$. These values are 3.4 and 7.4 times higher than those obtained for the RSR facility. The ratio of thermal to epithermal neutron fluence rates in the CT is $f=11$. This value is two times smaller than those observed for the RSR and indicates that neutron moderation increases with the distance from the core central position, as expected.

\section{Pneumatic Transfer Tube:}

The values obtained for thermal and epithermal fluence rates in the PTT are: $\phi$ th $=\left(2.5 \times 10^{11} \pm 8 \%\right) \mathrm{n} . \mathrm{cm}^{-2} \cdot \mathrm{s}^{-1}$ and $\phi$ epi $=\left(3.3 \times 10^{9} \pm 6 \%\right) \mathrm{n} . \mathrm{cm}^{-2} \cdot \mathrm{s}^{-1}$. The ratio of thermal to epithermal neutron fluence rate in the PTT is $f=75$, which is almost 5 times greater than the value obtained for the central tube.

Table 1 summarizes the results for neutron fluence rates in the IPR-R1 irradiation facilities.

TABLE I: IPR-R1 neutron fluence rates.

\begin{tabular}{cccc}
\hline Facility & $\begin{array}{c}\text { Thermal Neutron } \\
\text { Fluence Rate } \\
\left(\mathrm{n} . \mathrm{cm}^{-2} \cdot \mathrm{s}^{-1}\right) \times 10^{11}\end{array}$ & $\begin{array}{c}\text { Epithermal Neutron } \\
\text { Fluence Rate } \\
\left(\mathrm{n} . \mathrm{cm}^{-2} \cdot \mathrm{s}^{-1}\right) \times 10^{10}\end{array}$ & $\boldsymbol{f}$ \\
\hline RSR-5 & $8.0 \pm 1 \%$ & $3.5 \pm 1 \%$ & 23 \\
RSR-10 & $8.1 \pm 3 \%$ & $3.6 \pm 3 \%$ & 22 \\
RSR-15 & $8.4 \pm 1 \%$ & $3.6 \pm 1 \%$ & 23 \\
RSR-20 & $8.4 \pm 5 \%$ & $3.5 \pm 3 \%$ & 24 \\
RSR-25 & $8.3 \pm 1 \%$ & $3.5 \pm 1 \%$ & 24 \\
RSR-30 & $8.1 \pm 3 \%$ & $3.6 \pm 3 \%$ & 23 \\
RSR-35 & $8.1 \pm 1 \%$ & $3.3 \pm 1 \%$ & 24 \\
RSR-40 & $8.2 \pm 3 \%$ & $3.1 \pm 3 \%$ & 26 \\
RSR Average & $8.2 \pm 2 \%$ & $3.5 \pm 5 \%$ & 24 \\
\hline CT & $28.0 \pm 4 \%$ & $26.0 \pm 4 \%$ & 11 \\
PTT & $2.5 \pm 8 \%$ & $0.33 \pm 6 \%$ & 75 \\
\hline
\end{tabular}

\section{CONCLUSIONS}

In this work, thermal and epithermal neutron fluence rates were determined for the three irradiation facilities of the IPR-

\section{$\mathrm{R} 1$ reactor at the $\mathrm{CDTN} / \mathrm{CNEN}$.}

The mean values and standard deviations for the thermal and epithermal neutron fluence rates in the RSR are respectively $\phi_{t h}=\left(8.2 \times 10^{11} \pm 2 \%\right) n . \mathrm{cm}^{-2} \cdot \mathrm{s}^{-1}$ and $\phi_{e p i}=(3.5$ $\left.\times 10^{10} \pm 3 \%\right) \mathrm{n} \cdot \mathrm{cm}^{-2} \cdot \mathrm{s}^{-1}$, which correspond to $f=24$. The values obtained for the thermal and epithermal fluence rates in the CT central position are: $\phi_{t h}=\left(28.0 \times 10^{11} \pm 4 \%\right)$ n.cm ${ }^{-2} \cdot \mathrm{s}^{-1}$ and $\phi_{\text {epi }}=\left(2.6 \times 10^{11} \pm 4 \%\right) \mathrm{n} \cdot \mathrm{cm}^{-2} \cdot \mathrm{s}^{-1}$. The ratio of thermal to epithermal neutron fluence rate in the $\mathrm{CT}$ is $\boldsymbol{f}=11$. The values obtained for thermal and epithermal fluence rates in the PTT are: $\phi_{t h}=\left(2.5 \times 10^{11} \pm 8 \%\right)$ n.cm ${ }^{-2} \cdot \mathrm{s}^{-1}$ and $\phi_{\text {epi }}=\left(3.3 \times 10^{9} \pm 6 \%\right) \mathrm{n} \cdot \mathrm{cm}^{-2} \cdot \mathrm{s}^{-1}$. The ratio of thermal to epithermal neutron fluence rates in the PTT is $f=75$.

The results reveal that the epithermal neutron fluence rate in the PTT facility is approximately ten times smaller than that in the RSR which is about seven times smaller than that in the CT. In the case of the thermal neutron fluence rate, the rate ratio between the $\mathrm{CT}$ and the RSR or the RSR and the PTT is about three. These results show clearly the neutron thermalization from the center to the periphery of the reactor core, as expected. The $f$ parameter at the RSR is about two times higher than that for the CT, while for the PTT the $f$ value is about seven times higher than the $\mathrm{CT}$ value.

Knowledge of the thermal and epithermal neutron fluence rate in the irradiation facilities of the TRIGA IPR-R1is fundamental in the planning of sample irradiation for analysis or radioisotope production in a more accurate way. It will permit the calculation of the actual neutron reaction rates in the different irradiation channels, which will enable the determination of parameters such as specific induced activity, irradiation time, counting time, decay time, etc., for different experimental works. In a future article, the experimental values obtained in this work will be compared with results from a Monte Carlo simulation of IPR-R1 neutronic parameters.

\section{ACKNOWLEDGMENTS}

The authors would like to thank Dr. Amir Z. Mesquita, Fausto M. Júnior, M.Sc, supervisors of the TRIGA IPR-R1 reactor, as well as reactor operators Paulo F. Oliveira and Luiz O.I.S. Câmara. We are grateful to Geraldo F. Kastner, M.Sc, Dr. Rose M. G. P. Souza and Dr. Maria .B.C. Menezes for invaluable technical discussions.
[1] J.M. Lima, V.M. Andrade, Transactions of the American Nuclear Society 11(1), 270 (1968).

[2] H.M. Dalle, C. Pereira, R.G.P. Souza, Annals of Nuclear En- ergy 29, 901 (2002).

[3] D.M. Zangirolami, A.V. Ferreira, A.H. Oliveira, Brazilian Journal of Physics 39, 260 (2009). 
[4] M.Å.B.C. Menezes, R. Jacimovic, Nuclear Instruments \& Methods in Physics Research A 564, 707 (2006).

[5] K.H. Beckurts, "Neutron Physics", Springer-Verlag, New York, USA, (1964).

[6] Institute for Reference Materials and Measurements, Certified reference material IRMM-530R, Certificate of analysis, Gold mass fraction in Al-0.1\% Au Alloy. Geel, Belgium, (2007).
[7] F. de Corte, The $\mathrm{k}_{0}$-Standardization Method. A Move to the optimization of Neutron Activation Analysis, Thesis, Gent University, Belgium (1987).

[8] A.Z. Mesquita, H.C. Rezende, R. M. G. P. Souza, Proceedings of the 20th International Congress of Mechanical Engineering, Gramado, RS, Brazil, (2009). 